\title{
Features and Outcomes of Microcrystalline Arthritis Treated by Biologics: A Retrospective Study
}

\author{
Elise Doaré · François Robin · Hélène Racapé · Guillaume Le Mélédo • \\ Charles Orione · Pascal Guggenbuhl · Philippe Goupille • \\ Elisabeth Gervais · Emmanuelle Dernis · Béatrice Bouvard • \\ Thierry Marhadour · Guillaume Coiffier · Alain Saraux
}

Received: April 14, 2021 / Accepted: June 10, 2021 / Published online: July 4, 2021

(C) The Author(s) 2021

\section{ABSTRACT}

Objectives: The usual treatments for crystalassociated arthritis are sometimes contraindicated; thus, new therapies against interleukin1beta (IL-1) have been developed. We evaluated the characteristics of patients who received biological treatment for crystal-associated arthritis.

Details about the network "Société de Rhumatologie de l'Ouest and the network VICTOR HUGO" are available on https://www.srouest.fr/.

Supplementary Information The online version contains supplementary material available at https:// doi.org/10.1007/s40744-021-00335-7.

E. Doaré · G. Le Mélédo · T. Marhadour · A. Saraux Rheumatology Department, CHU, Centre de Référence Maladies Rares CERAINO, INSERM 1227, UBO, LabEx IGO, Brest CHU, Brest, France

F. Robin · P. Guggenbuhl · G. Coiffier Rennes, Service de Rhumatologie, Institut NUMECAN (Nutrition Metabolisms and Cancer), Univ Rennes, INSERM, INRA, 35000 Rennes, France

H. Racapé · B. Bouvard Rheumatology Department, CHU d'Angers, 49100 Angers, France

G. Le Mélédo · P. Goupille

Rheumatology Department, University Hospital of Tours; EA 7501, GICC, University of Tours, Tours, France
Patients and Methods: We conducted a multicentric retrospective observational study in six rheumatology units in western France. Patients receiving a biological treatment for crystal-associated arthritis between 1 January 2010 and 31 December 2018 were included. Improvement was defined as at least a $50 \%$ decrease in the count of synovitis and C-reactive protein level. Results: Forty-six patients were included: 31 (67.4\%) were treated for gouty arthritis, and 15 $(32.6 \%)$ for calcium pyrophosphate crystal deposition disease (CCPD). The first biotherapy used was anakinra for 14 patients (93.3\%) with CCPD and 31 patients (100.0\%) with gout. The first biotherapy course was more efficient in treating gout than in treating CCPD, with success in 28 patients $(90.3 \%)$ and 5 patients

\section{Orione}

Pneumology Department, CHU, Brest, France

\section{E. Gervais}

Rheumatology Department, CHRU de Poitiers, 86000 Poitiers, France

\section{E. Dernis}

Rheumatology Department, $\mathrm{CH}$, Le Mans, France

G. Coiffier

Rheumatology Unit, GHT Rance-Emeraude, CH, Dinan, France

\section{A. Saraux $(\square)$}

Rheumatology Unit, Hôpital de la Cavale Blanche, BP 824, 29609 Brest Cedex, France e-mail: alain.saraux@chu-brest.fr 
(35.7\%), respectively $(p=0.001)$. Six patients (42.9\%) with CCPD stopped their first biotherapy course because of side effects. Among the patients with gout, urate-lowering therapy was more frequently used after $(100 \%)$ than before the first biotherapy course $(67.7 \%)(p=0.002)$.

Conclusion: Anakinra was prescribed for cases of refractory crystal-associated arthritis or cases with contraindications for usual treatments. The efficacy of anakinra in treating CCPD was not obvious. Patients with CCPD had more side effects. The biotherapy was introduced with a long-term objective, while anti-IL-1 therapies are approved for acute crises only.

Keywords: Crystal-associated Biologic; Calcium pyrophosphate crystal deposition; Gout; Anti-IL-1

\section{Key Summary Points}

Anakinra is prescribed for cases of refractory crystal-associated arthritis or contraindications to usual treatments

The efficacy of anakinra in treating calcium pyrophosphate crystal deposition disease is not obvious

Anakinra is the most prescribed therapeutic, and its efficiency in treating gout was high

Anti-IL-1 therapies are generally introduced with a long-term objective

\section{DIGITAL FEATURES}

This article is published with digital features, including a summary slide, to facilitate understanding of the article. To view digital features for this article go to https://doi.org/10.6084/ m9.figshare.14762544.

\section{INTRODUCTION}

Crystal-associated arthritis is a major cause of inflammatory arthritis. Gout, calcium pyrophosphate crystal deposition disease (CCPD) and basic calcium phosphate crystal deposition disease (BCP) are the three main types of crystal-associated arthritis affecting adults [1]. The usual treatments rely on nonsteroidal anti-inflammatory drugs (NSAIDs), colchicine, and systemic or intra-articular glucocorticoids to reduce inflammation as well as pain [2-4]. However, in routine practice, contraindications are frequently seen because of the high prevalence of patient comorbidities [5].

It has been demonstrated that these types of crystal-associated arthritis have a common physiopathology and engage the caspase-1-activating NALP3 inflammasome, resulting in the production of active interleukin-1beta (IL-1beta) [6]. As a consequence, canakinumab, a fully human IL-1beta monoclonal antibody, has been studied in acute gouty arthritis [7]. In France, canakinumab has only been approved to treat frequent acute gouty arthritis when NSAIDs, colchicine and glucocorticoids are contraindicated, not tolerated or inefficient. Other IL-1beta monoclonal antibodies, such as anakinra, have also been evaluated and proven to be efficient in treating gouty arthritis [8]. Very few patients are treated with canakinumab, mostly because it is expensive. In addition, as crystal-associated arthritis and rheumatoid arthritis can be difficult to differentiate [9], some crystal-associated arthritis cases are treated using different biologic agents.

Consequently, a variety of biologics are used to treat crystal-associated arthritis without approval and routine practices can be heterogeneous. Thus, the aim of our study, the Features and evolution of Microcrystalline arthritis treated by BioLogics study (FUMBLE), was to evaluate the characteristics of patients who receive a biological treatment to treat crystalassociated arthritis, the therapeutic outcomes and the clinical course of treatment. 


\section{METHODS}

We conducted a multicentric retrospective observational study in adult patients (aged 18 years or more) receiving a biological treatment for crystal-associated arthritis. Patients were not included if they received a biologic treatment for another reason.

\section{Patients}

Patients treated in the rheumatology units of the hospitals in western France (Brest, Angers, Le Mans, Poitiers, Tours and Rennes) between 1 January 2010 and 31 December 2018 were identified. Patient selection was performed according to the availability of data in the corresponding centres of disease control:

- Patient databases in Brest, Rennes, Angers, Le Mans and Tours were searched in all categories (letters, keywords) with the following keywords: [microcrystalline disorder or crystal-associated arthritis or CCPD or hydroxyapatitis, or gouty arthritis] AND [Ilaris or canakimumab or anakinra or Kineret or anti-IL1 or anti-TNF or anti-IL-6].

- Poitiers does not use a database for research, and the patients followed in routine practice were included.

\section{Ethics}

This protocol 29BRC19.0056 was approved on 18 March 2019 by the ethics committee of Brest (B2019CE.14). A non-opposition letter was sent to each patient. This study was performed in accordance with the Helsinki Declaration of 1964 and its later amendments.

\section{Diagnosis}

The patients were screened according to the following crystal-associated arthritis criteria:

- For gouty arthritis, the ACR/EULAR 2015 criteria [10] were used, which means that gouty arthritis was confirmed by at least one episode of swelling, pain or tenderness in a peripheral joint or bursa associated with the presence of monosodium urate monohydrate crystal in a symptomatic joint, bursa or tophus. Otherwise, gouty arthritis was diagnosed when at least one episode of swelling in a peripheral joint or bursa associated with eight points according to the clinical, laboratory and imaging criteria occurred [10].

- For CCPD and BCP, the diagnosis was confirmed by at least one episode of swelling in a peripheral joint associated with corresponding crystals in the synovial fluid or radiographic features of CCPD/BCP deposition [11].

\section{Definition of Improvement and Treatment Efficacy}

Improvement was defined by at least a 50\% decrease in the swollen joint count and 50\% decreased in C-reactive protein (CRP) (if greater than $10 \mathrm{mg} / \mathrm{L}$ at inclusion) at the next visit after starting treatment. Patients with no swollen joint and no elevated CRP levels at the next visit after starting treatment were considered in remission. The time until next flare was not evaluated as it is difficult to define it in a retrospective study.

- We considered treatment efficient if the patient met the improvement or remission criteria.

\section{Collected Data}

For each selected patient, a physician completed a standardized case-report form by abstracting the epidemiological, clinical and radiological data from the medical charts.

\section{Statistical Analysis}

Descriptive analyses were performed using means ( \pm standard deviations, SDs) or medians ( \pm interquartile ranges, IQRs, i.e. Q1-Q3) for quantitative variables and percentages for categorical variables. Comparisons between 
populations were performed using the Wilcoxon nonparametric test for quantitative variables and the chi-square or Fisher's tests for categorical variables. Efficiency was compared using a paired Wilcoxon test for continuous variables and McNemar's test for categorical variables. Biotherapy duration, defined as the time between the establishment and termination of biotherapy, was evaluated using the Kaplan-Meier estimator. A difference with a $p$ value less than 0.05 was considered significant.

\section{RESULTS}

\section{Patient Characteristics}

With the database search, 925 patients from Tours, Le Mans, Angers, Rennes and Brest were identified. A total of 12 patients were excluded because they received biotherapy for another disease, 869 patients were excluded because they did not have crystal-associated arthritis or receive biotherapy, and 2 patients were excluded because the treatment was contraindicated; thus, we included 42 patients. Nine patients were from Brest, 19 were from Rennes, 7 were from Tours and 7 were from Angers/Le Mans. In addition, four patients from Poitiers were treated in routine practice and met the inclusion criteria. Overall, 46 patients were enrolled in this study (Fig. 1): 31 (67.4\%) were treated for gouty arthritis, and 15 (32.6) were treated for CCPD. No patients with BCP disease were included.

Table 1 shows the characteristics of the patients with crystal-associated arthritis. Five patients (33.3\%) with CCPD and 25 patients $(80.6 \%)$ with gout were male. The patients in the CCPD group were significantly older than were those in the gout group. Five of 10 patients $(50.0 \%)$ with CCPD and $18 / 21$ patients $(85.7 \%)$ with gouty arthritis had positive crystal fluid results. Renal function was statistically significantly different between groups, with a lower clearance in the gouty arthritis group $(p<0.001)$. Indeed, most patients with gouty arthritis had moderate renal failure. In both groups, patients frequently had cardiovascular disease such as ischemic heart disease, diabetes or arterial hypertension. Two patients with CCPD also had haemochromatosis. Rheumatoid factors and anti-citrullinated protein antibodies (ACPA) were found to be positive in 4/12 patients (33.3\%) and 3/12 patients (25.0\%) with CCPD disease and gout, respectively. We found a median swollen joint count of 3.50 (Q1-Q3 2.0-4.75) in the CCPD group and 4.0 (Q1-Q3 2.0-5.0) in the gout group before treatment. Seventeen patients $(45.9 \%)$ in the gout group

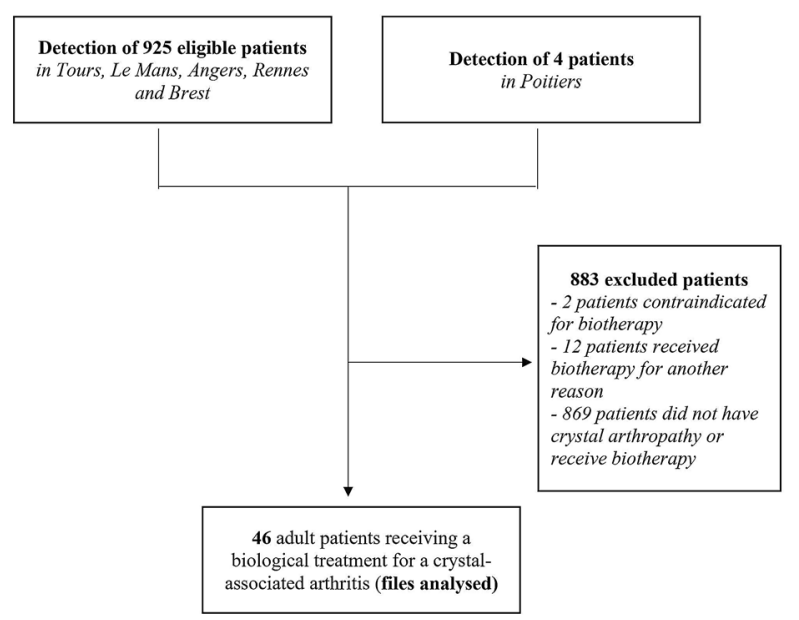

Fig. 1 Flow chart showing the patient selection process (performed by searching a database for any documents in all regions, except in Poitiers where the patients were included in routine practice) and the confirmation of cases 
Table 1 Characteristics of the patients with CCPD and gout treated by biologics in western France

\begin{tabular}{|c|c|c|c|}
\hline & CCPD & Gout & $p$ value \\
\hline Number & $15(32.6)$ & $31(67.4)$ & \\
\hline Sex female/total & $10(66.7)$ & $6(19.4)$ & 0.005 \\
\hline Age & $75.0(64.0-84.5)$ & $67.0(60.0-74.0)$ & 0.017 \\
\hline Weight & $84.0(73.0-87.0)$ & $85.0(76.15-93.0)$ & 0.139 \\
\hline Height & $157.0(154.0-171.5)$ & $173.5(164.2-176.5)$ & 0.041 \\
\hline Cellularity of synovial fluid & $700(300-14,000)$ & $13100(5750-22,500)$ & 0.150 \\
\hline Crystals in synovial fluid & $5 / 10(50.0)$ & $18 / 21(85.7)$ & 0.110 \\
\hline Uricaemia before treatment, $\mu \mathrm{mol} / \mathrm{L}$ & $310.0(246.5-369.5)$ & $527.0(432.0-623.0)$ & $<0.001$ \\
\hline Clearance, $\mathrm{mL} / \mathrm{min}$ & $81.15(78.05-88.0)$ & $54.0(34.25-69.25)$ & $<0.001$ \\
\hline CRP before treatment, $\mathrm{mg} / \mathrm{L}$ & $24.50(4.10-83.80)$ & $56.0(4.15-112.5)$ & 0.363 \\
\hline Positive RF $(n=27)$ & $4 / 12(33.3)$ & $1 / 15(6.6)$ & 0.021 \\
\hline Positive ACPA $(n=27)$ & $3 / 12(25.0)$ & $1 / 15(6.6)$ & 0.046 \\
\hline Positive ANA $>1 / 80(n=28)$ & $3 / 12(25.0)$ & $10 / 20(50.0)$ & 0.270 \\
\hline Arthritis before treatment & $3.50(2.0-4.75)$ & $4.0(2.0-5.0)$ & 0.755 \\
\hline Arthritis after treatment & $2.0(1.0-2.75)$ & $0.0(0.0-0.75)$ & 0.073 \\
\hline Tophus before treatment & $0(0.0)$ & $21(67.7)$ & $<0.001$ \\
\hline 1st MTP arthritis $(\geq 1)$ before treatment $(n=45)$ & $1 / 14(7.1)$ & $17 / 31(45.9)$ & 0.011 \\
\hline 1st MCP arthritis $(\geq 1)$ before treatment $(n=44)$ & $4 / 14(28.6)$ & $4 / 30(13.3)$ & 0.415 \\
\hline Hand arthritis $(\geq 1)$ before treatment $(n=46)$ & $10 / 15(66.7)$ & $14 / 31(45.2)$ & 0.327 \\
\hline Foot arthritis $(\geq 1)$ before treatment $(n=46)$ & $2 / 15(13.3)$ & $16 / 31(51.7)$ & 0.042 \\
\hline Knee arthritis $(\geq 1)$ before treatment $(n=43)$ & $6 / 14(42.9)$ & $18 / 29(62.1)$ & 0.238 \\
\hline \multicolumn{4}{|l|}{ Other arthritis before treatment } \\
\hline 0 & $5(33.3)$ & $22(71.0)$ & \\
\hline 1 & $6(40.0)$ & $4(12.9)$ & \\
\hline $2-3$ & $3(20.0)$ & $3(9.7)$ & \\
\hline$\geq 4$ & $1(6.7)$ & $2(6.5)$ & \\
\hline
\end{tabular}

Data are presented as $n(\%)$ or median $(\mathrm{Q} 1-\mathrm{Q} 3)$

$C C P D$ calcium pyrophosphate crystal deposition, $C R P$ C-reactive protein, $R F$ rheumatoid factor, $A C P A$ anti-citrullinated protein antibodies, $A N A$ antinuclear antibody, MTP metatarsophalangeal, $M C P$ metacarpophalangeal, Q1-Q3 quartile range 1-quartile range 3 
Table 2 First biotherapy and outcomes in CCPD and gout in western France

\begin{tabular}{|c|c|c|c|}
\hline & CCPD & Gout & $p$ value \\
\hline Number & $15(32.6)$ & $31(67.4)$ & \\
\hline First biotherapy & & & 0.708 \\
\hline Anakinra & $14(93.3)$ & $31(100.0)$ & \\
\hline Etanercept & $1(6.4)$ & 0 & \\
\hline Place of introduction & & & 0.497 \\
\hline Consultation & $4(26.7)$ & $13(41.9)$ & \\
\hline Hospitalization & $11(73.3)$ & $18(58.1)$ & \\
\hline Reason for introduction & & & 0.273 \\
\hline Crisis & $2(13.3)$ & $9(29.0)$ & \\
\hline Crisis/long-term & $3(20.0)$ & $9(29.0)$ & \\
\hline Long-term & $10(66.7)$ & $13(41.9)$ & \\
\hline Stopping reason & & & 0.048 \\
\hline Death & $0(0.0)$ & $2(6.5)$ & \\
\hline In progress & $3(20.0)$ & $5(16.1)$ & \\
\hline Side effect & $6(40.0)$ & $4(12.9)$ & \\
\hline Ineffective & $4(26.7)$ & $2(6.5)$ & \\
\hline Scheduled & $2(13.3)$ & $18(58.1)$ & \\
\hline Efficiency $(n=45)$ & $5 / 14(35.7)$ & $28 / 31(90.3)$ & $<0.001$ \\
\hline Side effect $(n=44)$ & $6 / 14(42.9)$ & $8 / 30(26.7)$ & 0.490 \\
\hline Stop for side effect $(n=44)$ & $6 / 14(42.9)$ & $4 / 30(13.3)$ & 0.084 \\
\hline
\end{tabular}

Data are presented as $n$ (\%)

$C C P D$ calcium pyrophosphate crystal deposition

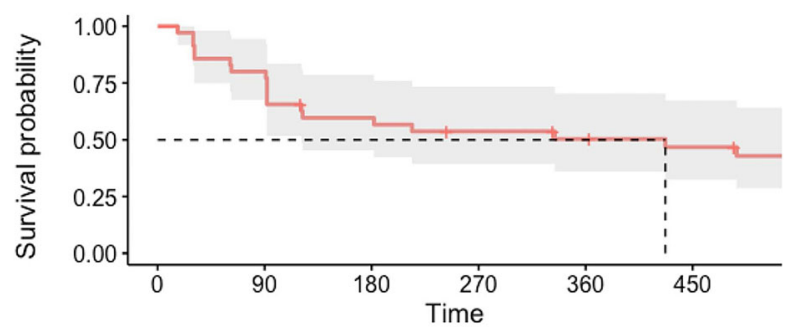

Fig. 2 Survival probability of biotherapy duration for long-term objective treatment 
Table 3 Evolution of medications, clinical and biological features after the 1st biotherapy course

\begin{tabular}{llll}
\hline $\boldsymbol{N}=\mathbf{4 6}$ & Before treatment & After treatment & $\boldsymbol{p}$ value \\
\hline Colchicine & $38 / 46(82.6)$ & $21 / 44(47.7)$ & $<0.001$ \\
NSAID & $9 / 44(20.5)$ & $6 / 44(13.6)$ & 0.505 \\
Oral corticosteroids & $30 / 45(66.7)$ & $21 / 45(46.7)$ & 0.016 \\
Local corticosteroids & $12 / 44(27.3)$ & $6 / 44(13.6)$ & 0.077 \\
Urate-lowering therapy & $22 / 46(47.8)$ & $31 / 45(68.9)$ & 0.008 \\
Arthritis & $4.0(2.0-5.0)$ & $0.0(0.0-2.0)$ & $<0.001$ \\
CRP & $39.0(4.0-97.4)$ & $5.0(1.8-9.0)$ & $<0.001$ \\
\hline
\end{tabular}

Data are presented as $n(\%)$ or median $(\mathrm{Q} 1-\mathrm{Q} 3)$

$N S A I D$ non-steroidal anti-inflammatory drug, CRP C-reactive protein

versus one $(7.1 \%)$ in the CCPD group had first metatarsophalangeal arthritis $(p=0.011)$.

\section{First Biotherapy Efficacy}

\section{Primary Outcome}

After the first biotherapy course, the patients had less severe arthritis. The first biotherapy course was more efficient in the gout group than in the CCPD group, with success in $28 / 31$ patients $(90.3 \%)$ and $5 / 14$ patients $(35.7 \%)$, respectively $(p=0.001) \quad$ (Table 2$)$. Fourteen patients $(93.3 \%)$ with CCPD and 31 patients (100.0\%) with gout received anakinra as the first biotherapy. It was introduced with the unique objective of long-term treatment in ten patients $(66.7 \%)$ with CCPD and 13 patients (41.9\%) with gout. Six patients (42.9\%) with CCPD and four patients $(13.3 \%)$ with gout stopped their first biotherapy course because of side effects. Biotherapy was more often discontinued in the gout group than in the CCPD group, with 18 patients $(58.1 \%)$ and 3 patients (20\%) in the gout and CCPD groups, respectively, discontinuing the therapy (Table 2). Among the 35 long-term-treated patients, 27 stopped their treatment because of side effects, a lack of efficiency, or death. The median duration of treatment was 427 days (95\% CI 121-731) (Fig. 2).

\section{Outcome}

Thirty-eight patients with gout (82.6\%) and 21 with CCPD (47.7\%) used colchicine before and after the first biotherapy course $(p<0.001)$. Thirty patients $(66.7 \%)$ received oral corticosteroids before and $21(46.7 \%)$ received the corticosteroids after biologic treatment $(p=0.0016)$. The use of local corticosteroids before (12 patients; $27.3 \%$ ) and after (6 patients; $13.6 \%)$ the first biotherapy course did not significantly differ $(p=0.077)$ but decreased by half. Urate-lowering therapy (ULT), such as allopurinol or febuxostat therapy, was more frequently used after the first biotherapy course (31 patients; $68.9 \%$ ) than before (22 patients; $47.8 \%)(p=0.008)$ (Table 3). The patients had less severe arthritis after the initiation of the first biotherapy course (median $=0$ ) than before. They also had a lower CRP level after treatment than before (median from 39.0 to $5.0 \mathrm{mg} / \mathrm{l})$. Among the gouty patients, allopurinol was more frequently used after the first biotherapy course (31 patients; 100\%) than before (21 patients; 67.7\%) ( $p=0.002)$ (Supplementary material 1$)$.

\section{Stratification by Centre}

There were no statistically significant differences among centres regarding the efficiency of the first biotherapy course $(p=0.055)$ (Table 4$)$. The duration of the first biotherapy course did not statistically significantly differ by centre 
Table 4 Stratification by centre

\begin{tabular}{lllllll}
\hline & Brest & Angers/Le Mans & Poitiers & Rennes & Tours & $\boldsymbol{p}$ value \\
\hline $\begin{array}{l}\text { Number } \\
\begin{array}{l}\text { 1st biotherapy } \\
\text { efficiency }\end{array}\end{array}$ & 9 & 7 & 4 & 19 & 7 & \\
$\begin{array}{l}\text { 1st biotherapy duration } \\
\text { (days) }\end{array}$ & 61.0 & 214.0 & $4 / 4(100.0)$ & $17 / 19(89.5)$ & $4 / 7(57.1)$ & 0.055 \\
$\begin{array}{l}\text { Reason for introduction } \\
\text { Crisis }\end{array}$ & $(26.0-92.0)$ & $(77.0-700.0)$ & $(216.0-1140.8)$ & $(120.5-699.5)$ & $(3.0-24.0)$ & \\
& $5(55.6)$ & $0(0.0)$ & $1(25.0)$ & $0(0.0)$ & $5(71.4)$ & $<$ \\
Crisis/long term & $0(0.0)$ & $1(14.3)$ & $0(0.0)$ & $11(57.9)$ & 0.057 \\
Long-term & $4(44.4)$ & $6(85.7)$ & $3(75.0)$ & $8(42.1)$ & $2(28.6)$ & \\
\hline
\end{tabular}

Data are presented as $n(\%)$ or median (Q1-Q3)

$(p=0.057)$, although the treatment duration seemed shorter in the Tours centre than in the other centres [3.0 days (Q1-Q3 3.0-24.0)]. In contrast, the reasons for introduction differed by centre $(p<0.001)$. Indeed, the centres in Rennes used biotherapy for both crises and long-term objectives in most cases (57.9\%) and never use biotherapy for a crisis only. The centres in Poitiers, Angers and Le Mans introduced biotherapy for long-term objectives, while the Tours centre used it mostly for crises (71.4\%). These results still hold when the data are stratified by centre and disease (Supplementary material 2). However, there were no significant differences in terms of crisis treatment between diseases or across centres (Supplementary material 3).

\section{Second Biotherapy Efficacy}

The patients received a second biotherapy course if the first one was inefficient or relapse occurred when the first biotherapy was introduced in the short term to treat a crisis.

Eight patients (17.4\%), five with CCPD and three with gout, received a second biotherapy course. Anakinra was used in three patients $(100.0 \%)$ with gout. A total of one patient (20.0\%) with CCPD received anakinra, one patient $(20.0 \%)$ received etanercept, one patient
(20.0\%) received infliximab and two patients (40.0\%) received abatacept. The second biotherapy course was efficient in one patient (20.0\%) with CCPD who was administered abatacept. No patients with CCPD receiving a different biotherapy than anakinra had positive ACPA or rheumatoid factor (RF). Side effects were found in one patient $(20.0 \%)$ with CCPD and two patients $(66.7 \%)$ with gout (Supplementary material 4).

\section{DISCUSSION}

To our knowledge, this is the first observational study focusing on the treatment of crystal-associated arthritis with all kinds of biotherapies in France.

Interestingly, the first biotherapy used was anakinra in $97.8 \%$ of the patients, and no patients were treated with canakinumab (despite its marketing authorisation in adult gout treatment in France in 2014). In addition, we observed that the first biotherapy was introduced with a long-term objective in 35 patients, while anti-IL-1beta therapies are currently approved for acute crises only in patients with gout. However, Solomon et al. [12] demonstrated that canakinumab can also prevent gout flares. Indeed, the authors randomized participants to groups receiving varying doses of 
canakinumab (50 mg, $150 \mathrm{mg}$ or $300 \mathrm{mg}$ ) or a placebo at 3-month intervals and performed follow-ups after a median of 3.7 years. The participants receiving canakinumab had a reduced risk for incident gout flares at all doses of canakinumab. This finding can explain why people were treated for a long period in our study, and the first biotherapy results were maintained in almost half of the patients at 450 days. However, it is important to note that all centres did not have the same therapeutic approach. Some centres, such as those in Angers, Le Mans and Rennes, tend to treat gout and CCPD with a long-term objective, while the Tours centre treated mostly crises with a short treatment duration. In routine practice, when patients are treated with a long-term objective, they receive anakinra once daily until remission for inflammation. Then, treatment is given less frequently if possible.

Interestingly, we found that among the patients with gout, the introduction of the first biotherapy course allowed the initiation of ULT in the short term. Indeed, $67.4 \%$ of the patients used ULT at baseline, whereas $100 \%$ use ULT after the initiation of the first biotherapy. It is well known that ULTs are the key treatment for gout because they prevent the occurrence of flares and complications of the disease by dissolving the pathogenic crystals [13]. However, in Europe, ULT is not started while patients have an acute gout attack because there is a risk of triggering the disease. We showed in our study that in difficult cases where usual treatments are inefficient or contraindicated, biotherapy can be an option to treat crises and introduce a ULT at the same time. A long biotherapy duration may also be linked to the fact that it is continued as a prophylactic treatment in some patients. Indeed, Schlesinger et al. [14] found in a randomized trial that a single canakinumab dose of at least $50 \mathrm{mg}$ or four weekly doses for 4 weeks provided superior prophylaxis against flares compared with daily colchicine $0.5 \mathrm{mg}$ in patients with gouty arthritis initiating allopurinol treatment.

After the first biotherapy course, the patients were less often treated with colchicine. However, there were no significant differences in the use of NSAIDs from before to after the first biotherapy course. Nevertheless, only $19.6 \%$ of patients took NSAIDs at baseline. Considering this small number, it is difficult to demonstrate a difference.

NSAIDs or colchicine are sometimes forbidden if the patient's level of renal function is too low. In our study, the patients with gout had a median clearance of $54.0 \mathrm{~mL} / \mathrm{min}$ (Q1-Q3 34.25-69.25). Loustau et al. [15] found that anakinra may be a safe therapeutic option for patients with gout and stage 4-5 chronic kidney disease or renal transplant. In addition, Yang et al. [16] showed that a single dose or scheduled adjustment was indicated for patients with severe renal impairment or end-stage renal disease when anakinra is used. Thus, it could be a good therapeutic option when NSAIDs or colchicine cannot be used.

A second biotherapy course was introduced in eight patients $(17.4 \%)$. Among the patients with CCPD, one $(20.0 \%)$ received anakinra, while four patients $(80.0 \%)$ did not receive an anti-IL-1 monoclonal antibody. However, only one patient $(20.0 \%)$ treated with abatacept showed improvement. None of these patients had positive ACPA or RF.

No patients had BCP disease in this study. Ea et al. [17] found in vivo that BCP crystal-induced synovitis was independent of IL- $1 \alpha$ and IL-1 $\beta$ signalling, and no changes in inflammation were observed in mice deficient for components of the NLRP3 inflammasome, IL- $1 \alpha$ or IL-1 $\beta$. In addition, treatment with anakinra did not prevent BCP crystal effects. This finding can explain why no studies on IL-1 inhibitors in BCP disease have been previously conducted and why no such patients were included in this study. However, Omoigui and Irene [18] reported that one patient with shoulder pain due to rotator cuff tendonitis and subacromial bursitis received subcutaneous injections of anakinra for 5 months. He showed improvement in the range motion of his shoulder in the 5th month and improvement in pain severity.

Canakinumab efficiency was evaluated in the b-RELIEVED trial [19]. The authors included a large sample size and reported that the mean $72 \mathrm{~h}$ visual analogue scale pain score was lower with canakinumab than with NSAIDs, with significantly less physician-assessed tenderness 
and swelling. Compared to NSAIDs, canakinumab significantly delayed the time to the first new flare and reduced the risk of new flares. Despite there being evidence of canakinumab being efficient in treating gout attacks, it seems that the cost $(11,361.39 €$ in France, for once every 3 months) may play an important role in the therapeutic decision. Anakinra has been proven to be efficient in treating gout flares in a randomized, noninferiority trial [20]. Fortythree patients received anakinra, and 45 received treatment as usual (NSAIDs or corticosteroids). The authors found that the efficiency of anakinra was noninferior to usual treatment in terms of pain. However, this is the only randomized clinical trial in patients with gout, and the lack of a large-scale study might explain why anakinra has not been approved to treat gout flare. Our results show that anakinra was efficient in $90.3 \%$ of patients with gouty arthritis, with a significant reduction in arthritis and CRP levels, confirming that this treatment can be a reasonable option to treat gout flares, with a better cost-effectiveness ratio than that of canakinumab (for a quarter the cost). New oral therapeutics against NLRP3 inflammasome are also being evaluated. Oral treatment such as dapansutrile could be an option to treat gout flares [21].

The efficacy of anakinra in treating CCPD was not obvious in this study. Indeed, we found that it was efficient in only $35.7 \%$ of the patients with CCPD, but treatment was frequently stopped because of adverse events. Compared with the number of gout treatment studies, there are relatively few studies investigating CCPD treatments, and currently, most treatments are based on clinical experience or conclusions extrapolated from the results of gout studies [22]. Indeed, only two studies reported the efficacy of anakinra in a retrospective study of 16 [23] and 33 [24] cases of CCPD arthritis. Hence, the most recent European recommendations do not include biotherapy as a possible option for CCPD crises, and neither anakinra nor canakinumab has been approved to treat CCPD in France. For example, the second biotherapy course was not as efficient in treating CCPD as was the first course, while it was effective in treating gout.
Even though IL-1 inhibitors can prevent and treat gout flares, the risk of infection has to be taken into account. Indeed, Schlesinger et al. [19] found more severe adverse events, such as infections or low neutrophil or platelet counts, in the canakinumab group than in the placebo group. In contrast, Liew and Gardner [25] assessed 29 patients who received anakinra for acute crystal arthritis in the presence of concurrent infection and did not observe any signs of the infections worsening that could be attributed to anakinra administration. In our study, we reported that $42.9 \%$ of the patients with CCPD and $13.3 \%$ of the patients with gout had to stop biotherapy because of side effects. In our study, the patients were treated for a longer period than were the patients in previous studies, and the patients with CCPD were older, which could explain the poor tolerance. Further investigations should be performed regarding safety, especially for long-term treatment. Our study has some limitations. First, this is a retrospective study. Thus, some relevant data might be missing. However, this is a real-world study, and ensuring that the practices were actually realized. In addition, to our knowledge no prospective study evaluated the efficiency of anakinra in CCPD. Second, the centres did not use the same procedure for patient selection. Indeed, the Poitiers centre does not use a database for research, so the patients who the physicians remembered fulfilled the inclusion criteria were included. Thus, with this method, some eligible patients may have been missed. However, considering the limited number of patients in each centre, it is highly improbable that a patient was forgotten. Third, few patients were included in this study, even though five centres were involved. However, Pascart et al. [26] investigated the proportion of patients with difficult-to-treat or difficult-to-prevent acute gout attacks eligible for IL-1 inhibition according to the current European guidelines in a French cohort. The authors found that despite the large number of patients without any firstline therapeutic options for gout flares, eligibility for IL-1 inhibition therapy was rare, and only $1 \%$ of the cohort was eligible. In France, gout prevalence was estimated to be $0.9 \%$ in the adult population in 2013 [27]. Thus, the small 
sample size of this study is in accordance with previous findings. Stratification by centre failed to demonstrate an effect on the efficiency and duration of the first biotherapy course. Fourth, patients with CCPD had a higher positive ACPA and RF count than in gout. Thus, some patients might have a rheumatoid arthritis and concurrently developed a CCPD disease. All patients with CCPD and ACPA or RF positive antibodies had at least radiographic signs of CCPD disease or a positive research result confirming crystal in the synovial fluid and standard arthritis localisation such as wrists or knees. Finally, probably many patients with osteoarthritis and asymptomatic elderly subjects with cartilage calcification should be included in this study as only $50 \%$ of the CCPD-arthritis group had evidence of synovial fluid crystals or a substantial proportion of CCPD-arthritis synovial fluid samples were non-inflammatory $(<2000$ cells/ $\mu \mathrm{L})$; and $33 \%$ were seropositive for $\mathrm{RF}(25 \%$ seropositive for CCP), and possibly had both CCPD and rheumatoid arthritis. The number of patients was too low to check whether the synovial fluid cell count was predictive of response to treatment.

\section{CONCLUSION}

Treatments for crystal-associated arthritis rely on symptomatic therapeutics associated with ULT in patients with gout. However, it might be difficult to treat acute attacks because of the presence of underlying comorbidities, such as hypertension, diabetes, renal insufficiency or gastrointestinal disorders, or the inefficiency of usual treatments. Without adequate treatment, patients may have persistent inflammation, which can contribute to joint destruction and impact quality of life. IL-1 inhibitors seem to be a good therapeutic option. However, homogeneous guidelines should be developed. Indeed, we showed that canakinumab, the only anti-IL1 authorised since 2014, is never used in clinical practice. Anakinra is the most prescribed therapeutic, and its efficiency in treating gout was high in this study. In addition, anti-IL-1 is supposed to be used for a short time to treat crises. However, we can see that in clinical practice, most patients receive long-term treatment for prophylaxis. Thus, additional studies should be carried out to clarify the role of anakinra in the therapeutic strategy.

\section{ACKNOWLEDGEMENTS}

We thank the participants of the study.

Funding. No funding or sponsorship was received for this study or publication of this article.

Authorship. All named authors meet the International Committee of Medical Journal Editors (ICMJE) criteria for authorship for this article, take responsibility for the integrity of the work as a whole, and have given their approval for this version to be published.

Authorship Contributions. Elise Doaré collected the data and drafted the study. François Robin, Hélène Racapé and Guillaume Le Mélédo collected the data. Charles Orione and Alain Saraux performed the statistical analysis. Pascal Guggenbuhl, Philippe Goupille, Elisabeth Gervais, Emmanuelle Dernis, Béatrice Bouvard, Thierry Marhadour, Guillaume Coiffier revised this study critically for important intellectual content. Alain Saraux conceived and supervised the findings of this study. All authors reviewed the results and approved the final version of the manuscript.

Disclosures. Elise Doaré, François Robin, Hélène Racapé, Guillaume Le Mélédo, Charles Orione, Pascal Guggenbuhl, Philippe Goupille, Elisabeth Gervais, Emmanuelle Dernis, Béatrice Bouvard, Thierry Marhadour, Guillaume Coiffier and Alain Saraux have nothing to disclose.

Compliance with Ethics Guidelines. The study was conducted in accordance with the Good Clinical Practice guidelines and Medical Ethics Committee guidelines. This protocol 29BRC19.0056 was approved on 18 March 2019 by the ethics committee of Brest (B2019CE.14). This study was performed in accordance with the Helsinki Declaration of 1964 and its later 
amendments. All patients gave their consent to the physician who signed a non-opposition document.

Data Availability. All data generated or analysed during this study are included in this published article/as supplementary information files. The datasets generated during and/or analysed during the current study are available from the corresponding author on reasonable request.

Open Access. This article is licensed under a Creative Commons Attribution-NonCommercial 4.0 International License, which permits any non-commercial use, sharing, adaptation, distribution and reproduction in any medium or format, as long as you give appropriate credit to the original author(s) and the source, provide a link to the Creative Commons licence, and indicate if changes were made. The images or other third party material in this article are included in the article's Creative Commons licence, unless indicated otherwise in a credit line to the material. If material is not included in the article's Creative Commons licence and your intended use is not permitted by statutory regulation or exceeds the permitted use, you will need to obtain permission directly from the copyright holder. To view a copy of this licence, visit http://creativecommons.org/licenses/by$\mathrm{nc} / 4.0 /$.

\section{REFERENCES}

1. Choi H. Epidemiology of crystal arthropathy. Rheum Dis Clin North Am. 2006;32(2):255-73. https://doi.org/10.1016/j.rdc.2006.03.002 (v).

2. Richette P, Doherty M, Pascual E, et al. 2016 updated EULAR evidence-based recommendations for the management of gout. Ann Rheum Dis. 2017;76(1):29-42. https://doi.org/10.1136/ annrheumdis-2016-209707.

3. Khanna D, Khanna PP, FitzGerald JD, et al. 2012 American College of Rheumatology guidelines for management of gout part II: therapy and anti-inflammatory prophylaxis of acute gouty arthritis. Arthritis Care Res. 2012;64(10):1447-61. https:// doi.org/10.1002/acr.21773.
4. Latourte A, Pascart T, Flipo R-M, et al. 2020 Recommendations from the French Society of Rheumatology for the management of gout: management of acute flares. Joint Bone Spine. 2020;87(5):387-93. https://doi.org/10.1016/j. jbspin.2020.05.001.

5. Keenan RT, O'Brien WR, Lee KH, et al. Prevalence of contraindications and prescription of pharmacologic therapies for gout. Am J Med. 2011;124(2): 155-63. https://doi.org/10.1016/j.amjmed.2010.09. 012.

6. Martinon F, Pétrilli V, Mayor A, Tardivel A, Tschopp J. Gout-associated uric acid crystals activate the NALP3 inflammasome. Nature. 2006;440(7081): 237-41. https://doi.org/10.1038/nature04516.

7. Schlesinger N, De Meulemeester M, Pikhlak A, et al. Canakinumab relieves symptoms of acute flares and improves health-related quality of life in patients with difficult-to-treat gouty arthritis by suppressing inflammation: results of a randomized, dose-ranging study. Arthritis Res Ther. 2011;13(2):R53. https://doi.org/10.1186/ar3297.

8. Ottaviani S, Moltó A, Ea H-K, et al. Efficacy of anakinra in gouty arthritis: a retrospective study of 40 cases. Arthritis Res Ther. 2013;15(5):R123. https://doi.org/10.1186/ar4303.

9. Nunes EAS, Rosseti AG, Ribeiro DS, Santiago M. Gout initially mimicking rheumatoid arthritis and later cervical spine involvement. Case Rep Rheumatol. 2014. https://doi.org/10.1155/2014/ 357826.

10. Neogi T, Jansen TLTA, Dalbeth N, et al. 2015 Gout classification criteria: an American College of Rheumatology/European League Against Rheumatism collaborative initiative. Arthritis Rheumatol Hoboken NJ. 2015;67(10):2557-68. https://doi.org/ 10.1002/art.39254.

11. Devauchelle-Pensec V, Berthelot JM, Jousse S, et al. Performance of hand radiographs in predicting the diagnosis in patients with early arthritis. J Rheumatol. 2006;33(8):1511-5.

12. Solomon DH, Glynn RJ, MacFadyen JG, et al. Relationship of interleukin-1 $\beta$ blockade with incident gout and serum uric acid levels: exploratory analysis of a randomized controlled trial. Ann Intern Med. 2018;169(8):535-42. https://doi.org/ 10.7326/M18-1167.

13. Pascart T, Latourte A, Flipo R-M, et al. recommendations from the French Society of Rheumatology for the management of gout: urate-lowering therapy. Joint Bone Spine. 2020. https://doi.org/10. 1016/j.jbspin.2020.05.002. 
14. Schlesinger N, Mysler E, Lin H-Y, et al. Canakinumab reduces the risk of acute gouty arthritis flares during initiation of allopurinol treatment: results of a double-blind, randomised study. Ann Rheum Dis. 2011;70(7):1264-71. https://doi.org/10.1136/ard. 2010.144063 .

15. Loustau C, Rosine N, Forien M, et al. Effectiveness and safety of anakinra in gout patients with stage 4-5 chronic kidney disease or kidney transplantation: a multicentre, retrospective study. Joint Bone Spine. 2018;85(6):755-60. https://doi.org/10.1016/ j.jbspin.2018.03.015.

16. Yang B-B, Baughman S, Sullivan JT. Pharmacokinetics of anakinra in subjects with different levels of renal function. Clin Pharmacol Ther. 2003;74(1): 85-94. 9236(03)00094-8.

17. Ea H-K, Chobaz V, Nguyen C, et al. Pathogenic role of basic calcium phosphate crystals in destructive arthropathies. PLoS ONE. 2013;8(2): e57352. https://doi.org/10.1371/journal.pone.0057352.

18. Omoigui S, Irene S. Subcutaneous injection of anakinra in patients with shoulder pain due to rotator cuff tendonitis and subacromial bursitis. Pain Med Malden Mass. 2004;5(2):229-30. https:// doi.org/10.1111/j.1526-4637.2004.04022.x.

19. Schlesinger N, Alten RE, Bardin T, et al. Canakinumab for acute gouty arthritis in patients with limited treatment options: results from two randomised, multicentre, active-controlled, double-blind trials and their initial extensions. Ann Rheum Dis. 2012;71(11):1839-48. https://doi.org/ 10.1136/annrheumdis-2011-200908.

20. Janssen CA, Oude Voshaar MAH, Vonkeman HE, et al. Anakinra for the treatment of acute gout flares: a randomized, double-blind, placebo-controlled, active-comparator, non-inferiority trial. Rheumatol Oxf Engl. 2019. https://doi.org/10. 1093/rheumatology/key402.
21. Klück V, Jansen TLTA, Janssen M, et al. Dapansutrile, an oral selective NLRP3 inflammasome inhibitor, for treatment of gout flares: an open-label, dose-adaptive, proof-of-concept, phase 2 a trial. Lancet Rheumatol. 2020;2(5):e270-80. https://doi. org/10.1016/S2665-9913(20)30065-5.

22. Zhang W, Doherty M, Pascual E, et al. EULAR recommendations for calcium pyrophosphate deposition. Part II: management. Ann Rheum Dis. 2011;70(4):571-5. https://doi.org/10.1136/ard. 2010.139360 .

23. Ottaviani S, Brunier L, Sibilia J, et al. Efficacy of anakinra in calcium pyrophosphate crystal-induced arthritis: a report of 16 cases and review of the literature. Joint Bone Spine. 2013;80(2):178-82. https://doi.org/10.1016/j.jbspin.2012.07.018.

24. Thomas M, Forien M, Palazzo E, Dieudé P, Ottaviani S. Efficacy and tolerance of anakinra in acute calcium pyrophosphate crystal arthritis: a retrospective study of 33 cases. Clin Rheumatol. 2019;38(2): 425-30. https://doi.org/10.1007/s10067-018-42722 .

25. Liew JW, Gardner GC. Use of anakinra in hospitalized patients with crystal-associated arthritis. J Rheumatol. 2019;46(10):1345-9. https://doi.org/ 10.3899/jrheum.181018.

26. Pascart T, Norberciak L, Ea H-K, Graf S, Guggenbuhl P, Lioté F. Difficult-to-treat gout flares: eligibility for interleukin-1 inhibition in private practice is uncommon according to current EMA approval. Rheumatol Oxf Engl. 2019;58(12):2181-7. https:// doi.org/10.1093/rheumatology/kez203.

27. Bardin T, Bouée S, Clerson P, et al. Prevalence of gout in the adult population of France. Arthritis Care Res. 2016;68(2):261-6. https://doi.org/10. 1002/acr.22660. 